Teoria Quântica: Uma Abordagem Quantizada da sua Evolução - Parte I

\author{
Félix, F. K. C.; Bonfim, P. G. B.; Santos, L. S. S.; Pereira, D. H*
}

Rev. Virtual Quim., 2016, 8 (2), 319-337. Data de publicação na Web: 8 de fevereiro de 2016

http://www.uff.br/rvq

\title{
Quantum Theory: An Approach Quantized on its Evolution - Part I
}

Abstract: The Interest in the interaction of electromagnetic radiation with matter was the first impulse to structure a series of formulas, postulates and theories that would become the foundation for quantum physics. Under the shadow of statistical determinism, in the XIX and XX centuries, started the studies on the blackbody radiation, a consequent reformulation of various theories of classical physics and the introduction of the concepts of energy quantization, radiation and particles. This article will deal, briefly and chronologically, with the evolution of the old quantum theory.

Keywords: Atomic models; photoelectric effect; Blackbody radiation.

\section{Resumo}

O interesse pela interação da radiação eletromagnética com a matéria foi o primeiro impulso para a estruturação de uma série de fórmulas, postulados e teorias que seriam o alicerce para a física quântica. Sob a sombra do determinismo estatístico, nos séculos XIX e XX, iniciaram-se os estudos sobre a radiação do corpo negro, uma consequente reformulação de várias teorias da física clássica e a introdução da quantização de energia, radiação e partículas. O presente artigo abordará, de forma resumida e cronológica, a evolução da antiga teoria quântica.

Palavras-chave: Modelos atômicos; efeito fotoelétrico; radiação do corpo negro.

\footnotetext{
* Universidade Federal do Tocantins, Campus Universitário de Gurupi, Rua Badejós, Lote 7, Chácaras 69/72, Zona Rural, CEP 77402-907, Gurupi-TO, Brasil.

Mdoug@uft.edu.br

DOI: $10.5935 / 1984-6835.20160024$
} 


\title{
Teoria Quântica: Uma Abordagem Quantizada da sua Evolução - Parte I
}

\author{
Fernanda K. C. Félix, Pedro G. B. Bonfim, Lucas S. S. Santos, Douglas \\ Henrique Pereira*
}

Universidade Federal do Tocantins, Grupo de pesquisa em Química Teórica, Campus Universitário de Gurupi, Rua Badejós, Lote 7, Chácaras 69/72, Zona Rural, CEP 77402-907, Gurupi-TO, Brasil.

* doug@uft.edu.br

Recebido em 23 de julho de 2015. Aceito para publicação em 4 de fevereiro de 2016

\section{Introdução}

\section{Da problemática do corpo negro à introdução do termo quanta}

2.1. Wilhelm Wien

2.2. Ludwig Planck

\section{Dos quantas de luz ao equilíbrio térmico da radiação}

\subsection{Albert Einstein}

3.2. Niels Bohr

3.3. Sommerfeld

4. Da equação de onda do elétron ao cálculo de sua energia e momento relativísticos

\section{Conclusão}

\section{Introdução}

A Mecânica Newtoniana fortaleceu uma antiga concepção determinista, ${ }^{1}$ que descrevia o conhecimento do observador sobre as condições iniciais de um sistema e das equações diferenciais que regem a evolução deste, como meio para prever suas características em qualquer instante tanto do futuro quanto do passado. ${ }^{2} \mathrm{Um}$ dos maiores defensores do determinismo foi Pierre $\mathrm{S}$. Laplace, que considerava que o estado presente do universo seria um efeito dos seus estados passados e causa para seus estados futuros. Laplace supôs uma inteligência que pudesse conhecer todas as forças que agiriam sobre um sistema e pudesse submeter os dados coletados pela rápida observação a uma análise matemática. Para tal inteligência nada seria incerto, pois o futuro e o passado estariam presentes ante seus olhos. ${ }^{3}$

Posteriormente, a inteligência descrita por Laplace como meio de estabelecer matematicamente o futuro e o passado de 
uma situação, foi denominada de "Demônio de Laplace". ${ }^{2}$ Muitos autores consideram inexata a definição laplaciana de determinismo, pois nela só era considerado o sistema que tivesse suas condições iniciais precisamente determinadas. ${ }^{1}$

O demônio de Laplace foi aos poucos ficando doente, e um dos grandes agravantes desta doença foi a perda da causalidade, muito defendida por Leibniz. ${ }^{2}$ Enquanto isto o indeterminismo crescia, se firmando nas noções de probabilidade, que advinha da falta de conhecimento sobre alguns dos efeitos causais envolvidos na análise de certa situação. Surge então, como meio de tangenciar esta dificuldade de descrever um fenômeno através das carências de suas causas, o determinismo estatístico.

Boltzmann, um dos criadores da mecânica estatística, auxiliou na consolidação do trabalho de Maxwell sobre a distribuição de velocidades e equipartição de energia para o caso geral de partículas. ${ }^{4}$

Maxwell interpretava a função de distribuição de velocidades como uma densidade de probabilidade, enquanto Boltzmann a interpretava como uma fração de tempo, de um intervalo suficientemente longo, durante o qual a velocidade de uma molécula se manteria dentro de certos limites. Com o objetivo de provar a unicidade da distribuição de Maxwell para descrever estados de equilíbrio termodinâmico, ${ }^{4}$ Boltzmann derivou uma equação para a evolução da função da distribuição de velocidades, e mostrou que a distribuição de Maxwell era uma solução estacionária da equação, e que depois de obtida, ela não se alteraria. ${ }^{5}$

A influência exercida por Boltzmann para a física moderna, em particular, para a mecânica quântica, é nomeável. Ele usou a noção de quantização de energia já em 1872 ao dividir a energia de um sistema em pequenos pacotes e assim permitir o uso de equações de combinação de probabilidades, e através deste método considerou a entropia de um sistema em um estado proporcional à probabilidade daquele estado. $^{6,7}$

A teoria cinética dos gases de Maxwell e Boltzmann fortaleceu as interações entre a Mecânica e a Termodinâmica, e exerceu forte impacto sobre Planck e Einstein, principalmente a respeito das pesquisas sobre a radiação do corpo negro, ${ }^{7,8}$ quantum de luz ${ }^{9,10}$ e movimento browniano. ${ }^{6,11}$

Como a ciência é a organização estruturalmente lógica dos fatos que a compõem, vê-se a necessidade de abordar de maneira consistente e cronológica os fatos que levaram ao desenvolvimento da teoria quântica. ${ }^{12}$ Tal abordagem é aqui feita através dos cientistas que trabalharam para a sua estruturação. Começando por Kirchhoff, Wien, Boltzmann e Planck, e seus trabalhos sobre a radiação do corpo negro e a consequente introdução da ideia de quantum de energia em $1900,8,13$ e indo até De Broglie, com a elaboração de sua tese sobre ondas de matéria em 1924, ${ }^{14-16}$ finalizando assim o período que é conhecido como antiga teoria quântica, e consequentemente do determinismo estatístico.

A necessidade desta revisão bibliográfica se apoia no fato da má estruturação de vários conceitos fundamentais de química, que são expostos aos alunos de Graduação de maneira confusa, diminuindo o interesse destes por esta área. Este artigo trará uma nova abordagem das proposições que formaram e constituem a antiga teoria quântica e apresentará resumida e cronologicamente os fatos e fórmulas que alicerçam todo o conhecimento acerca da quantização de energia, com o propósito de se tornar futuramente, fonte de pesquisa aos alunos interessados na história da física/química quântica.

\section{Da problemática do corpo negro à introdução do termo quanta}

\subsection{Wilhelm Wien}


Dos vários caminhos que a teoria quântica poderia ter seguido, o mais aceito pelos físicos, químicos e historiadores é que esta surgiu através de estudos sobre as propriedades térmicas da radiação do corpo negro, ${ }^{13}$ consolidando-se em 1900. Para melhor entendimento do desenvolvimento da teoria quântica é necessária uma retrospectiva dos fatos que levaram a ela, partindo das tentativas mais notáveis de descrição dos corpos negros.

$$
u=\frac{4 \Phi}{c}
$$

Em que $\square$ representa a densidade de radiação, $\square$ representa fluxo de energia emitida pelo orifício e $\square$ é uma constante relacionada às dimensões da cavidade.

Vinte anos depois, em 1879, o físico Josef Stefan constatou que a densidade total de
Um corpo, após absorver energia, normalmente calor, emite radiação eletromagnética com intensidade variável. $^{7}$ Corpos que absorvem completamente a radiação eletromagnética que nele incide são denominados corpos negros. ${ }^{17}$ Conforme o Teorema de Kirchhoff, um corpo negro ideal é uma cavidade com um pequeno orifício, pelo qual a radiação pode entrar e sair livremente. Esta relação é dada pela seguinte fórmula:

Eq. 1

energia do corpo negro é proporcional à quarta potência da temperatura. ${ }^{17}$ Boltzmann, em 1884, demonstrou este resultado utilizando a teoria eletromagnética de Maxwell; para isto ele representou o fluxo de um corpo negro como: ${ }^{7}$

$$
F=\int_{0}^{\infty} F(v) d v=\int_{0}^{2 \pi} d \varphi \int_{0}^{\pi / 2} \cos \theta \operatorname{sen} \theta d \theta \int_{0}^{\infty} u(v, T) d v=\pi \int_{0}^{\infty} u(v, T) d v
$$

Assim, a intensidade específica frequências pode ser representada por: monocromática para todo o espectro de

$$
\int_{0}^{+\infty} u(\mathrm{v}, T) d \mathrm{v}=\sigma T^{4} \quad \text { Eq. } 3
$$

Considerando $\square$ a temperatura e $\square$ a constante Stefan-Boltzmann, com valor de $5,67 \times 10^{-8} \mathrm{~W} / \mathrm{m}^{2} \mathrm{~K}^{4}$.

Um dos cientistas que mais influenciou a descrição dos fenômenos que envolviam um

$$
\lambda_{\operatorname{MAX}} \propto \frac{1}{T}
$$

Outro importante resultado dos estudos de Wien sobre as propriedades do corpo negro foi a lei de deslocamento que permite a determinação espectral para qualquer temperatura, uma vez conhecida a sua distribuição. $^{7}$ corpo negro foi Wilhelm Wien. Sua lei de 1896 dizia que o comprimento de onda $\square$ correspondente à intensidade máxima no espectro é inversamente proporcional à temperatura absoluta do corpo: ${ }^{8}$

Eq. 4

Entretanto a determinação da forma funcional $f\left(\frac{v}{T}\right)$ tornou-se um dos maiores problemas para a física teórica no final do século XIX. ${ }^{7}$ Como tentativa para tal determinação, em 1896, Wien propôs que: 


$$
\begin{array}{ll}
p(v, T)=v^{3} f\left(\frac{v}{T}\right) & \text { Eq. } 5 \\
f\left(\frac{v}{T}\right)=\alpha \exp \left(-\beta \frac{v}{T}\right) & \text { Eq. } 6
\end{array}
$$

Sendo $\alpha$ e $\beta$ constantes, e mesmo sem possuir fundamento teórico suficiente, a Eq. (5) ajustava-se aos resultados experimentais.

Em junho de 1900, Lord Rayleigh, propôs uma nova lei para o espectro do corpo negro.

$$
E=\left(\frac{R}{N}\right) T
$$

Sendo que $R$ é a constante dos gases perfeitos (8.314472 $\mathrm{J} / \mathrm{mol} . \mathrm{K}), N$ o número de Avogrado $\left(6.0221415 \times 10^{23}\right.$ moléculas $\left./ \mathrm{mol}\right)$ e $\frac{R}{N}$ a constante de Boltzmann $\left(1,3806503 \times 10^{-23}\right.$

$$
u d \lambda=\left(\frac{8 \pi R T}{N \lambda^{4}}\right) d \lambda
$$

Lord Rayleigh acrescentou ao final do artigo em que apresentou as Eqs. (7) e (8), que era necessária a introdução de um fator exponencial de correção para completar a lei de distribuição derivada e em 1902 declarou que a lei só seria válida na faixa das baixas frequências (longos comprimentos de onda). ${ }^{17}$

\subsection{Ludwig Planck}

É neste cenário em que entram os trabalhos do físico Ludwig Planck, que inicialmente discordava da mecânica estatística de Boltzmann, sendo para ele, a Segunda Lei da Termodinâmica uma verdade absoluta. ${ }^{12}$ Ao considerar o Teorema de Kirchhoff, que postulava que a radiação possuía um caráter universal, ${ }^{7}$ e seguir o alerta de Boltzmann, de que era necessária a introdução de uma hipótese adicional às leis
Utilizando a teoria clássica de ondas, ele estabeleceu que a densidade de ondas obedece à relação $\mathrm{N}_{\lambda}=\left(\frac{8 \pi}{\lambda^{4}}\right)$ e que cada onda estaria associada a uma energia $E$, conforme a Eq. (7):

\section{Eq. 7}

$\mathrm{J} / \mathrm{K})$, naquela época ainda não conhecida por este nome, e hoje dada pelo símbolo $k_{B}$.

Conforme Rayleigh, a densidade de energia obedeceria a seguinte equação:

Eq. 8

reversíveis da Termodinâmica, ${ }^{17}$ Planck foi forçado a explicar a irreversibilidade dos fenômenos radiativos utilizando novos conceitos e leis alheios à Termodinâmica e Eletrodinâmica, naquela época consideradas completamente fundamentadas e verdadeiras.

Na busca por explicar como a radiação e a matéria interagem para atingir um equilíbrio termodinâmico, sem lançar mão de hipóteses outras que não fossem as da Termodinâmica e da Eletrodinâmica, Planck considerou que a radiação eletromagnética interatuasse com um conjunto de osciladores harmônicos, denominados de ressonadores, que agiria como um modelo simplificado de matéria. ${ }^{8}$ Conforme Kichhoff havia mostrado, há uma independência entre a distribuição de radiação de um corpo negro e a composição da matéria. ${ }^{7,8}$

Assim, em 1899, Planck considerou algumas leis clássicas do Eletromagnetismo, 
como a densidade da radiação elétrico $\left(E_{x}\right)$ em ondas planas: ${ }^{8}$ eletromagnética $\left(p_{v}\right)$ e a expansão do campo

$$
\begin{array}{ll}
p_{v}=\frac{3}{2 \pi \tau}|f(v)|^{2} & \text { Eq. } 9 \\
E_{x}=\int_{-\infty}^{+\infty} d v f(v) \exp (2 \pi i v t) & \text { Eq. } 10
\end{array}
$$

E calculou a direção $(x(t))$ e a frequência inicial $\left(v_{0}\right)$ de um ressonador de massa $(m)$ :

$$
\begin{array}{ll}
x(t)=\frac{q}{2 \pi v_{0} m} \int d t^{\prime} E_{x}\left(t^{\prime}\right) \sin \left[2 \pi v_{0}\left(t-t^{\prime}\right)\right] & \text { Eq. } 11 \\
v_{0}=\frac{1}{2 \pi} \sqrt{\frac{k}{m}} & \text { Eq. } 12
\end{array}
$$

Sabendo que a potência de um ressonador é dada pela Eq. (13), Planck relacionou essa equação com a densidade da radiação eletromagnética $\left(u_{v}\right)$, obtendo a Eq. (14).

$$
\begin{aligned}
& \delta P_{v}=\frac{q}{\tau} \int d t^{\prime} \dot{x} E_{x} \\
& \delta P_{v}=\frac{\pi q^{2}}{3 m} p_{v}
\end{aligned}
$$

Para um caso generalizado, a potência dos ressonadores pode ser expressa como:

$$
P_{\text {ress }}=\frac{\pi q^{2}}{3 m} p(v, T)
$$

Considerando que as médias da potência da radiação $(P)$ e energia $(\bar{u}(v, T))$ de um ressonador devido à sua oscilação são representadas respectivamente por: ${ }^{8}$

$$
\begin{aligned}
& P=\frac{2 q^{2}}{3 c^{3}}(2 \pi v)^{2} \overline{x^{2}} \\
& \bar{u}(v, T)=m(2 \pi v)^{4} \overline{x^{2}}
\end{aligned}
$$

Planck obteve a fórmula para potência da radiação: 
Félix, F. K. C. et al.

$$
P_{\text {rad }}=\frac{2 q^{2}}{3 c^{3}}(2 \pi v)^{2} \bar{u}(v, T)
$$

E igualando as Eqs. (15) e (18), Planck obteve uma expressão que relaciona a densidade de energia $p(v, T)$ com a energia média do conjunto de osciladores

$$
p(v, T)=\frac{8 \pi}{c^{3}} v^{2} \bar{u}(v, T)
$$

Este resultado auxilia na compreensão das propriedades da radiação eletromagnética emitida por diversos corpos, além do corpo negro. ${ }^{7}$

A partir da Eq. (19) e dos conhecimentos sobre os trabalhos de Wien demonstrados

$$
S=-\frac{u}{\beta v} \ln \left(\frac{u}{A \exp (v)}\right)
$$

Sendo $u$ a energia média dos osciladores harmônicos e $A=\frac{\alpha c^{3}}{8 \pi}$.

No início de 1900, Lummer e Pringsheim mediram a radiação entre os comprimentos de onda $12 \mu \mathrm{m}$ e $18 \mu \mathrm{m}$ a temperaturas entre $300 \mathrm{~K}$ e $1.650 \mathrm{~K}$, enquanto uma segunda equipe composta por Rubens e Kurlbaum varreu os comprimentos de onda entre 30 $\mu \mathrm{m}$ e $60 \mu \mathrm{m}$ a temperaturas entre $200 \mathrm{~K}$ e $1500 \mathrm{~K}$, concluindo, ambas as equipes, que a fórmula Wien não era válida para comprimentos de onda mais longos dentro de uma longa faixa de temperatura. ${ }^{7,8,10}$

Era o início de uma nova pesquisa, em que físicos teóricos e experimentais procuravam fórmulas que descrevessem a radiação para todos os comprimentos de onda do espectro. Entretanto, essa busca era complicada; alguns pesquisadores chegaram a considerar que a radiação comportava-se de maneira diferente nos longos e curtos comprimentos harmônicos $\bar{u}(v, T)$ que representam os átomos na superfície do corpo negro em equilíbrio termodinâmico:

Eq. 19

pelas Eqs. (6) e (7) acerca da radiação do corpo negro, Planck obteve a entropia do sistema formado pelo equilíbrio termodinâmico entre os ressonadores e a radiação, que, por não ser decrescente, obedece à segunda lei da termodinâmica: ${ }^{8}$

Eq. 20

de onda. Caso isto fosse verdade o comportamento da radiação já estaria completamente descrito pelas equações de Rayleigh e Wien.

No dia 7 de outubro de 1900, após a visita de Heinrich Rubens, Planck ficou ciente de que para longos comprimentos de onda, a densidade de energia era proporcional à temperatura, ${ }^{9,10}$ o que o auxiliou a encontrar, nesta mesma noite, sua fórmula da radiação, representada na Eq. (24), ${ }^{7}$ que foi comunicada à Academia Alemã de Física no dia 19 de outubro, mesmo dia em que foi apresentado que os resultados experimentais do comportamento da radiação em longos comprimentos de onda não eram compatíveis com as equações de Wien. ${ }^{9}$

Para propor a Eq. (24), Planck considerou que a entropia dos osciladores poderia ser dada por:

$$
\frac{\partial^{2} S}{\partial u^{2}}=-\frac{1}{u(\alpha+u)}
$$


Integrando a expressão anterior e considerando $\frac{\partial S}{\partial u}=\frac{1}{T}$, tem-se:

$$
\frac{1}{\alpha} \ln \left(\frac{\alpha+u}{u}\right)+c=\frac{1}{T}
$$

Sendo $c=0$, a energia do oscilador é fornecida pela equação:

$$
u=\frac{\alpha}{\exp (\alpha / T)-1}
$$

Utilizando a Eq. (19) e observando a lei de deslocamento de Wien, Planck verificou que $\alpha$ seria uma função linear de $v$, e assim, a Eq. (23) foi reescrita:

$$
p(v, T)=\frac{8 \pi}{c^{3}} \frac{A v^{3}}{\exp (B v / T)-1}
$$

Planck não pronunciou, em momento algum, que o resultado de Rayleigh tenha influenciado em seu trabalho, ${ }^{8}$ apesar de que ele provavelmente tivesse conhecimento dos artigos de Rayleigh que foram publicados na influente Philosophical Magazine. ${ }^{7,17}$

Em seus trabalhos, Planck mostrou que o caminho mais correto para a formulação de uma teoria a respeito do espectro de radiação térmica seria a determinação teórica da entropia em função da energia de um oscilador harmônico, ${ }^{7}$ entretanto, ele chegou a esta conclusão utilizando derivações da fórmula de Wien, que falha

$$
u=\frac{\varepsilon}{\exp \left(\varepsilon / k_{B} T\right)-1}
$$

Ao aplicar a lei do deslocamento de Wien, Planck percebeu que o elemento de energia $\varepsilon$

$$
\varepsilon=h v
$$

para longos comprimentos de onda. Caso a Eq. (24) estivesse correta, o que foi confirmado pelos experimentos de Lummer e Pringsheim, Planck poderia derivá-la a partir de uma expressão para a entropia. ${ }^{13}$ Para tal fim, viu-se obrigado a desfazer-se de seu preconceito sobre a mecânica estatística e considerar a relação entre entropia e probabilidade, seguindo assim, um caminho já trilhado por Boltzmann. ${ }^{8}$

No transcorrer de seus cálculos, Planck define a fórmula que descreve a energia média dos osciladores:

Eq. 25

deveria ser proporcional ao número de oscilações de $v$, sendo: ${ }^{10}$

E assim: 


$$
p(v, T)=\frac{8 \pi}{c^{3}} \frac{h v^{3}}{\exp \left(h v / k_{B} T\right)-1}
$$

Por fim, Planck encontrou os valores das duas constantes naturais presentes em sua fórmula, com ajuda de medidas já disponíveis, sendo elas denominadas de: constante de Planck $h=6,6260755 \times 10^{-34}$ J.s e constante de Boltzmann $k_{B}=$ $1,380658 \times 10^{-23} \mathrm{~J} \mathrm{~K}^{-1}$. $^{10}$ Estes resultados foram apresentados à Sociedade de Física no dia 14 de dezembro de 1900, quando Planck declarou que a energia não é contínua. ${ }^{9}$

No decorrer do tempo, e com o advindo das pesquisas de Einstein, Planck passou por uma mudança radical tanto em seu vocabulário como em seu modo de pensar, adotando o termo quantum, para o elemento de energia $\varepsilon$, e admitindo sua descontinuidade. ${ }^{17}$

\section{Dos quantas de luz ao equilíbrio térmico da radiação}

\subsection{Albert Einstein}

A discussão sobre a constituição da luz vinha sendo travada desde o século XVII, com Isaac Newton e Robert Hooke (que defendiam a teoria corpuscular para a luz) e Christiaan Huygens (que defendia a teoria ondulatória da luz). ${ }^{18}$ Por um longo tempo, sobreviveu a teoria de Isaac Newton, até que a hipótese ondulatória voltou a ter força com o inglês Thomas Young. Entretanto esta teoria não conseguia descrever a intensidade de radiação luminosa emitida por um corpo negro. E com o desafio lançado por Gustav Kirchhoff, em 1896, de formular uma teoria que descrevesse com precisão o comportamento do corpo negro, os pilares e experimentos que comprovavam a teoria ondulatória da luz estavam abalados. ${ }^{19}$

Max Planck, após rever seus conceitos tratou a energia de forma não contínua, conseguindo assim estabelecer uma equação que descrevesse a radiação luminosa que saía de um corpo negro limitando a descontinuidade da energia à emissão dos osciladores do corpo negro. ${ }^{13}$

Albert Einstein, em seu trabalho de 1905, "Um ponto de vista heurístico sobre a produção e transformação da luz", introduziu uma hipótese que generalizou as características dos osciladores de Planck para a própria radiação. Assim a energia não estaria distribuída de maneira contínua, mas por um número finito de quantas de energia localizado em pontos do espaço. ${ }^{18}$ Desta maneira, a teoria corpuscular da luz ressurgia diferente daquela imaginada por Newton. ${ }^{20}$ Neste mesmo artigo, em que Einstein discorreu sobre a quantização do campo de radiação, ${ }^{21}$ também propôs uma explicação para o que ficou conhecido como efeito fotoelétrico. Conforme a teoria clássica do eletromagnetismo, a energia cinética do elétron deveria aumentar com a intensidade da luz, mas observava-se, pelo efeito fotoelétrico, que a emissão dos elétrons devido à incidência de luz aumentava não com a intensidade da fonte incidente, mas com a frequência. ${ }^{19}$

Ao estudar o efeito fotoelétrico, Einstein percebeu que a energia cinética do elétron ejetado era igual à diferença da energia do quantum de luz $(h v)$ e a profundidade do poço de potencial. Assim, o elétron só seria ejetado se possuísse certa energia cinética $K$, o que implicaria em uma frequência de corte como descrito na Eq. (28): ${ }^{22}$

$$
K=h v-W
$$


Em que $K$ é a energia cinética do elétron, $h$ é a constante de Planck, $v$ é a frequência e $W$ é a função do trabalho, que seria a energia requerida para remover os elétrons do metal. Esta dependência linear entre energia e frequência foi confirmada experimentalmente dez anos depois, através do trabalho de Millikan na Universidade de Chicago e de Duane e Hunt em Harvard. ${ }^{16}$

$$
K=h v=m c^{2}=p c
$$

Em que $p$ é o momento do fóton e $m$ é a sua massa.

$$
p=\frac{h}{\lambda}
$$

O conceito dualístico da luz foi introduzido a partir da Eq. (30), ou seja, a luz possui comprimento de onda e momento. ${ }^{16}$ Este conceito foi uma das primeiras demonstrações do futuro princípio da complementaridade do físico dinamarquês Niels Bohr, em que qualquer teoria quântica deveria se aplicar também às teorias clássicas. ${ }^{16,19,21}$ Niels Bohr, em 1913 - munido de ideias como o movimento orbital do

$$
\frac{1}{\lambda}=R_{y}\left(\frac{1}{n_{f}^{2}}-\frac{1}{n_{i}^{2}}\right)
$$

Onde: $n_{i}>n_{f}, \lambda$ é o comprimento de onda correspondente à linha espectral em centímetros e $R_{y}$ é a constante de Rydberg, com valor de $109677 \mathrm{~cm}^{-1}$.

De acordo com a Física Clássica, quando o elétron estivesse girando em torno de um

$$
\frac{m \mathrm{v}^{2}}{r}=\frac{Z e^{2}}{4 \pi \varepsilon_{0} r^{2}}
$$

Em que $m$ e $e$ são a massa e a carga do elétron respectivamente, $Z$ é a carga do

\subsection{Niels Bohr}

Baseando-se em sua Teoria da Relatividade, Einstein, sugeriu que a energia cinética do fóton fosse dada pela expressão:

\section{Eq. 29}

A partir da Eq. (29) e considerando o comprimento de onda da luz equivalente à $\lambda=\frac{c}{v}$, Einstein obteve:

Eq. 30

elétron, quantização, e utilizando a fórmula de Rydberg e a espectroscopia atômica, reformulou o modelo atômico de seu professor Ernest Rutherford. ${ }^{23,24}$

Para a formulação de seu modelo atômico, Bohr analisou o átomo de hidrogênio, que é simples e possui seu espectro completamente descrito pela Eq. (31) de Rydberg. ${ }^{25}$

\section{Eq. 31}

núcleo, atuaria sobre ele uma força centrípeta que seria igual à força eletrostática Coulombiana, ${ }^{23}$ pois neste caso o movimento orbital do elétron seria como o movimento acelerado de uma carga elétrica. ${ }^{23}$ Assim:

\section{Eq. 32}

núcleo, $\varepsilon_{0}$ a permissibilidade do vácuo, v é a velocidade e $r$ é o raio do orbital. Através Rev. Virtual Quim. |Vol 8| |No. 2| |319-337| 
desta equação encontram-se os valores potencial $V$ : correspondentes de energia cinética $K \mathrm{e}$

$$
\begin{aligned}
& K=\frac{1}{2} \frac{Z e^{2}}{4 \pi \varepsilon_{0} r} \\
& V=-\frac{Z e^{2}}{4 \pi \varepsilon_{0} r}
\end{aligned}
$$

Sendo a energia mecânica a soma dessas duas energias, então:

$$
E=-\frac{Z e^{2}}{2 r 4 \pi \varepsilon_{0}}
$$

A frequência da radiação corresponde à:

$$
v=\frac{\mathrm{v}}{2 \pi r}
$$

O elétron em seu movimento orbital perderia energia de maneira contínua, ${ }^{23}$ esta perda satisfaria variações do tipo:

$$
\Delta \mathrm{E}(2,1)=E(2)-E(1)=\frac{Z e^{2}}{2 r 4 \pi \varepsilon_{0}(1)}-\frac{Z e^{2}}{2 r 4 \pi \varepsilon_{0}(2)}
$$

Desta maneira, com a perda de energia, o raio orbital tornar-se-ia cada vez menor enquanto a frequência de radiação aumentaria. Este processo terminaria na colisão do elétron com o núcleo atômico, ${ }^{23}$ o que não é observado experimentalmente. Sabe-se que o átomo é estável e que seu espectro de emissão não é contínuo e sim discreto.

Compreendendo que os átomos irradiam certas frequências definidas apenas ao serem

$$
h v_{b a}=E_{b}-E_{a}
$$

Sendo $E_{b}$ a energia do estado inicial e $E_{a}$ a energia no estado final (após a emissão).

Considerando que a energia $E$ é descrita pela Eq. (35), seria necessário quantizar o excitados, Bohr postulou que os elétrons se movem em certas órbitas sem irradiar energia. Nesta situação, os elétrons estariam em estados estacionários.

A irradiação de energia ocorreria apenas quando o elétron sofresse uma transição de um estado estacionário para outro, e a frequência da radiação emitida estaria relacionada à energia das órbitas. Esta energia emitida durante a transição corresponderia à: ${ }^{26}$

Eq. 38

raio da órbita, pois de acordo com a equação, o raio seria a única variável contínua. Com o propósito de obter o raio quantizado, Bohr postulou que o momento orbital angular do 
elétron era quantizado em múltiplos de $\hbar$, assim:

$$
r p=n \hbar
$$

Eq. 39

De tal modo, o momento linear do elétron seria:

$$
L=m v r=\frac{n h}{2 \pi}=n \hbar
$$

Isolando o valor da velocidade temos que:

$$
\mathrm{v}=\frac{n \hbar}{m r}
$$

Ao substituir v na Eq. (32), Bohr obteve o raio quantizado, também chamado de Raio de Bohr, $a_{0}$ :

$$
a_{0}=\frac{4 \pi \varepsilon_{0} n^{2} \hbar^{2}}{m Z e^{2}}
$$

Nas Eqs. (39), (40) e (41), $m, v$ e $r$ correspondem à massa, velocidade e raio do elétron respectivamente, $n$ é o número quântico principal, $Z$ refere-se ao número atômico e $e$ à carga do elétron. Atribuindo valores numéricos para o raio de Bohr, $\hbar=$ $1,054 \times 10^{-34} \mathrm{~J} . \mathrm{s} ; e=1,602 \times 10^{-19} \mathrm{C} ; m=9,109$ $\times 10^{-31} \mathrm{Kg} ; \varepsilon_{0}=8,854 \times 10^{-12} \mathrm{C}^{2} \mathrm{~J}^{-1} \mathrm{~m}^{-1} ; \mathrm{Z}=1$ e $\mathrm{n}$

$$
\begin{aligned}
& E=-\frac{m Z^{2} e^{4}}{2 n^{2} \hbar^{2}\left(4 \pi \varepsilon_{0}\right)^{2}} \\
& v=\frac{1}{2 \pi} \frac{m Z^{2} e^{4}}{n^{3} \hbar^{3}\left(4 \pi \varepsilon_{0}\right)^{2}}
\end{aligned}
$$

$=1$ obtemos o valor de $a_{0}=5,29 \times 10^{-11} \mathrm{~m}$ para o raio do átomo de hidrogênio. Os valores correspondentes às constantes supracitadas foram obtidos da referencia 27.

A energia e a frequência de radiação poderiam então ser obtidas substituindo o valor do Raio de Bohr nas Eqs. (35) e (36):
A partir da equação de energia de Bohr podemos encontrar a equação Eq. (31) de Rydberg. ${ }^{25}$ : Dada a Eq. (26) em que a energia
Eq. 43

Eq. 44

elementar é dada em termos de $h v$, a energia em um sistema em transição pode ser expressa como:

$$
\Delta \mathrm{E}=h v
$$

Eq. 45

Como $v=c / \lambda$, essa equação pode ser reescrita: 
Félix, F. K. C. et al.

$$
\Delta \mathrm{E}=h \frac{C}{\lambda}
$$

Isolando $1 / \lambda$ tem-se:

$$
\frac{1}{\lambda}=\frac{\Delta E}{h c}
$$

Substituindo o valor de $\Delta E$ encontrado no modelo de Bohr, obtemos a equação de Rydberg:

$$
\frac{1}{\lambda}=R_{y}\left(\frac{1}{n_{f}^{2}}-\frac{1}{n_{i}^{2}}\right)
$$

Em que:

$$
R_{y}=\frac{m e^{4}}{8 \varepsilon_{0}^{2} h^{3} c}
$$

Atribuindo os valores numéricos para as constantes na Eq (48) encontramos o valor $109737 \mathrm{~cm}^{-1}$,que é próximo ao valor encontrado experimentalmente por Rydberg. O modelo atômico de Bohr encontrava algumas limitações por não explicar espectros de átomos mais complexos que o hidrogênio, e carecer de informações sobre as intensidades das linhas espectrais e a divisão de certas raias. ${ }^{16,25}$ O físico alemão, Sommerfeld, em 1916, ao notar que as órbitas de um átomo eram degeneradas, possuindo desta forma a mesma energia total, e que essa energia dependia somente do número quântico principal $n$, compreendeu que era necessário introduzir um novo número quântico ao modelo atômico de Bohr. ${ }^{28}$

\subsection{Sommerfeld}

Na tentativa de explicar a estrutura fina do espectro do hidrogênio, Sommerfeld introduziu órbitas elípticas ao modelo de Bohr, e descreveu o movimento em termos das coordenadas polares $r$ e $\theta$, aplicando duas condições de quantização e de estabilidade mecânica:

$$
\begin{aligned}
& \oint L d \theta=n_{\theta} h \quad \therefore L=n_{\theta} \hbar \\
& \oint p_{r} d r=n_{r} h \therefore L\left(\frac{a}{b}-1\right)=n_{r} \hbar
\end{aligned}
$$

A partir destas condições, Sommerfeld calculou os semieixos maior $(a)$ e menor $(b)$ das órbitas elípticas e a energia total $(E)$ de um elétron nessa órbita.

$$
a=\frac{4 \pi \epsilon_{0} n^{2} \hbar^{2}}{\mu Z e^{2}}
$$




$$
\begin{aligned}
& b=\frac{a n_{\theta}}{n} \\
& E=-\left(\frac{1}{4 \pi \epsilon_{0}}\right) \frac{\mu Z^{2} e^{4}}{2 n^{2} \hbar^{2}}
\end{aligned}
$$

Em que o valor $\mu$ corresponde à massa do elétron e $n$ corresponde ao número quântico principal, que indica o número de diferentes órbitas possíveis e que é definido por:

$$
n=n_{\theta}+n_{r}
$$

Sendo $n_{\theta}$ o número quântico azimutal.

Como os estados de um átomo são degenerados, para um dado valor de $n$, há

$$
\mu=\mu_{0} \gamma \therefore \frac{\mu_{0}}{\sqrt{\frac{1-v^{2}}{c^{2}}}}
$$

Em que $\mu_{0}$ é a massa de repouso e $v$ é sua velocidade.

Assim, Sommerfeld demonstrou que a energia dependia dos números quânticos principal $n$ e azimutal $n_{\theta}$, obtendo a
Eq. 52

várias órbitas diferentes, que são especificadas pelo momento angular quantizado, Eq. (40). Para estas órbitas a energia total seria idônea. Para tangenciar a degenerescência do átomo de hidrogênio, Sommerfeld utilizou a Teoria da Relatividade Restrita, apresentada por Einstein em 1905. Desta forma, ele descreveu a massa efetiva do elétron, quando este se encontra em uma velocidade aproximadamente igual à da luz.

\section{Eq. 54}

separação das linhas espectrais, e formulando uma equação que apresentava estados degenerados e que satisfazia perfeitamente a Eq. (43), de Bohr. ${ }^{29}$

$$
E=-\left(\frac{1}{4 \pi \epsilon_{0}}\right) \frac{\mu Z^{2} e^{4}}{2 n^{2} \hbar^{2}}\left[1+\frac{\alpha^{2} Z^{2}}{n}\left(\frac{1}{n_{\theta}}-\frac{3}{4 n}\right)\right] \quad \text { Eq. } 55
$$

Onde $\alpha$ é a constante de estrutura fina (linhas espectrais muito próximas entre si e que geralmente só são vistas em espectroscópios sofisticados), ${ }^{16}$ com valor de $\frac{e^{2}}{4 \pi \epsilon_{0} \hbar c}$.

A Eq. (55) foi verificada ainda em 1916, por Frederich Paschen, em um experimento utilizando hélio ionizado. ${ }^{30}$

A partir de 1916, Einstein começou a questionar o comportamento da radiação ao atingir o equilíbrio térmico, e em sua teoria inseriu os conceitos de emissão espontânea e emissão estimulada. Para o desenvolvimento desta teoria, ele empregou o novo conceito de estados estacionários introduzido por Niels Bohr em 1913. ${ }^{21,26}$ Einstein procurou explicar, em seu artigo de 1917, as propriedades da radiação, considerando que o os átomos possuíssem dois níveis de energia $a$ e $b$, sendo $E_{b}>E_{a}$, ou seja, $a$ é um estado de menor energia que $b$. Os estados seriam ocupados de acordo com a distribuição de Boltzmann com probabilidades: 


$$
\begin{aligned}
& P_{a}=C \exp \left(\frac{E_{a}}{k T}\right) \\
& P_{b}=C \exp \left(\frac{E_{b}}{k T}\right)
\end{aligned}
$$

Em que $C$ é um fator de normalização, $k$ é a constante de Boltzmann e $T$ é a temperatura.

Utilizando a lei de deslocamento de Wien, obtida a partir da Eq. (5), Einstein notou três processos pelos quais os átomos interagem

$$
d W=A_{b a} d t
$$

E $A_{b a}$ é uma constante.

A radiação absorve energia de um campo de força com um espectro amplo com uma taxa que é proporcional à densidade espectral do campo. Ao absorver energia, o

$$
d W=p(v, T) B_{a b} d t
$$

Em que $B_{a b}$ é uma constante.

A emissão estimulada ocorre quando o campo de radiação de um átomo no estado de energia superior faz com que ocorra uma

$$
d W=p B_{b a} d t
$$

Em que $B_{b a}$ é uma constante.

Considerando as condições para o equilíbrio térmico e a lei de deslocamento de Wien, Einstein mostrou que $B_{b a} \propto A_{b a}$ e que $B_{b a}=B_{a b} \cdot{ }^{21}$ Ao fazer uma transição, o átomo emite uma radiação monocromática com frequência dada pela condição de Bohr, Eq. (44).

Admitindo que os átomos estejam em equilíbrio térmico, Einstein deduziu as propriedades do campo de radiação com a radiação: emissão espontânea, absorção e emissão estimulada.

A emissão espontânea ocorre quando um átomo excitado faz uma transição para um estado mais baixo de energia, ou seja, $b \rightarrow a$. A probabilidade de que esta emissão ocorra em um tempo $d t$ é: ${ }^{21}$

\section{Eq. 58}

átomo será excitado, e o elétron se deslocará para um estado mais alto de energia, ou seja, $a \rightarrow b$, a probabilidade de que isto ocorra em um intervalo de tempo $d t$ é: ${ }^{21}$

\section{Eq. 59}

transição para um estado inferior, com uma taxa proporcional à densidade de radiação. $\mathrm{A}$ probabilidade de fazer uma transição $b \rightarrow a$ no tempo $d t$ é: ${ }^{21}$

\section{Eq. 60}

necessárias para manter este equilíbrio, e obteve precisamente a Lei de Radiação de Planck, Eq. (27). ${ }^{21}$ 


\section{Da equação de onda do elétron ao cálculo de sua energia e momento relativísticos}

Em 1924, o príncipe francês, Louis de Broglie, $^{14}$ lembrando-se da natureza dualística dos fótons, sugerida por Einstein na Eq. (30), e considerando que os fenômenos naturais que envolviam a matéria e a radiação fossem semelhantes, sugeriu haver ondas de matéria, ou seja, as partículas que, como o elétron, por exemplo, possuiriam um momento $p$ e seriam associadas a uma onda de comprimento $\lambda^{16}$

Muitos livros didáticos e artigos argumentam, com o objetivo de abordar superficialmente a teoria de Broglie, que ele chegou a sua conclusão sobre ondas de matéria, igualando e manipulando as Eqs. (26) e (29). ${ }^{31}$ Entretanto, esta igualdade,

$$
\psi_{0}=A \operatorname{sen} 2 \pi\left(v_{0} t_{0}\right)
$$

Saindo do repouso, perde-se a sincronia do fenômeno periódico devido a efeitos relativistas. ${ }^{15}$ Como todas as leis válidas da física deveriam permanecer inalteradas sobre representada na Eq. (61), leva a uma contradição, pois o aumento da velocidade acarreta a diminuição da frequência e o aumento da massa. ${ }^{15}$

$$
E=m c^{2}=h v \quad \text { Eq. } 61
$$

A fim de evitar esta contradição, de Broglie analisou o elétron como um sistema infinito, ao considerar o princípio do eletromagnetismo de Maxwell que diz que a energia de qualquer carga está espalhada no espaço à sua volta, embora haja uma forte concentração no centro. ${ }^{15}$ A partir de pressuposições físicas, de Broglie chegou à Eq. (62), em que o elétron em repouso tem toda sua estrutura pulsando em sincronia, e sua frequência seria dada pela Eq. (61).

\section{Eq. 62}

uma transformada de Lorentz, ${ }^{31}$ de Broglie a aplicou sobre a Eq. (62), substituindo a variável tempo $t$, por:

$$
t=\left(t^{\prime}+\frac{v x^{\prime}}{c^{2}}\right) \gamma
$$

$$
\begin{aligned}
& \text { Em que } \gamma=\frac{1}{\sqrt{1-\frac{v^{2}}{c^{2}}}} \text { obtendo: } \\
& \qquad \psi_{0}=A \operatorname{sen} 2 \pi\left[v_{0} \frac{1}{\sqrt{1-\beta^{2}}}\left(t-\frac{\beta x}{c}\right)\right]
\end{aligned}
$$

A Eq. (64) é uma equação de uma onda monocromática movendo-se na direção do eixo $x$. Desta maneira, de Broglie demonstrou que a oscilação, quando relacionada a outros referenciais, transformava-se em uma onda. Obteve ainda a frequência, a velocidade e outras propriedades desta onda. ${ }^{15}$

No propósito de obter a energia e o momento relativísticos, de Broglie utilizou a massa relativística efetiva da partícula, expressa na Eq. (54), e considerando que a 
partícula move-se a uma velocidade $\mathrm{v}$, momento linear: ${ }^{31}$ reescreveu a Eq. (54) em termos de seu

$$
p=\frac{m_{0} \mathrm{v}}{\sqrt{1-\frac{\mathrm{v}^{2}}{c^{2}}}}
$$

Através de manipulações nesta equação, substituindo $\frac{1}{\sqrt{1-\frac{v^{2}}{c^{2}}}}$ por $\gamma$ e utilizando $m=m_{0} \lambda$, obteve-se:

$$
p^{2} c^{2}=-m_{0}^{2} c^{4}+\frac{m^{2}}{\gamma^{2}} c^{4} \gamma^{2} \therefore p c=\sqrt{\left(m c^{2}\right)^{2}-m_{0}^{2} c^{4}} \quad \text { Eq. } 66
$$

Onde $p c$ é o momento do elétron (partícula).

De acordo com a correlação de Einstein, obtém-se a energia relativística: ${ }^{31}$

$$
E=m c^{2}=\sqrt{p^{2} c^{2}+m_{0}^{2} c^{4}}
$$

A teoria de de Broglie, de que os elétrons comportam-se como onda, foi comprovada em 1927, nos laboratórios da Bell Co. por Davisson e Germer, em um experimento em que uma superfície de cristral de níquel foi bombardeada por um feixe de elétrons de baixa energia. Ao fazer isso eles observaram que o comportamento dos elétrons foi semelhante ao dos raio $X$ em relação ao ângulo de espalhamento e, utilizando a equação de Bragg $(n \lambda=2 d \operatorname{sen} \theta)$, calcularam para o elétron um comprimento de onda, conforme o previsto por de Broglie. ${ }^{16}$

\section{Conclusão}

As sessões anteriores fazem uma viagem pela história, no compromisso de relatar a evolução da Antiga Teoria Quântica. Iniciando-se pelos acontecimentos marcantes do final do século XIX e início do XX. Desde o desafio lançado por Kirchoff, de que seria fundamental para a Física descrever o comportamento da radiação emitida por um corpo negro, às tentativas de físicos ilustres como Stefan, Boltzmann, Wien, Rayleigh, e finalmente Planck, que ao formular novos termos e quantizar a energia dos ressonadores, conseguiu, em 1900, chegar a uma equação que satisfizesse o desafio de Kirchoff. Baseado nas ideias de Planck, Einstein elaborou sua explicação sobre o efeito fotoelétrico e sobre o comportamento da radiação quando em equilíbrio térmico; Bohr reformulou o modelo atômico de Rutherford introduzindo orbitas quantizados; Sommefeld complementou o modelo de Bohr ao considerar orbitais degenerados $e$ elípticos; e de Broglie, relembrando dos postulados de Einstein, introduziu o conceito de ondas de matéria, abrindo caminho para uma nova época da física, para o fim o determinismo estatístico e o início do indeterminismo, em que novos físicos reescrevem a história. 


\section{Agradecimentos}

Os autores gostariam de agradecer o suporte financeiro do $\mathrm{CNPq}$ (Conselho Nacional de Desenvolvimento Científico e Tecnológico), CAPES e o professor Rogério Custodio pelas contribuições.

\section{Referências Bibliográficas}

${ }^{1}$ Silveira, F. L. Determinismo, previsibilidade e caos. Caderno Catarinense do Ensino de Física 1993, 10, 137. [Link]

${ }^{2}$ Ryckman, T. Why history matters to philosophy of physics. Studies in History and Philosophy of Science Part A 2015, 50, 4. [CrossRef]

${ }^{3}$ Bordoni, S. On the borderline between Science and Philosophy: A debate on determinism in France around 1880. Studies in History and Philosophy of Science Part A 2015, 49, 27. [CrossRef]

${ }^{4}$ Dahmen, S. R. Ludwig Boltzmann: vida e obra. Gazeta de Física 2007, 30, 16. [Link]

${ }^{5}$ Brading, K. Physically locating the present: A case of reading physics as a contribution to philosophy. Studies in History and Philosophy of Science Part A 2015, 50, 13. [CrossRef]

${ }^{6}$ Cercignani, C.; Ludwig Boltzmann: the man who loved atoms, Oxford Universit Press: Oxford, 1998. [Link]

${ }^{7}$ Studart, N. A invenção do conceito de quantum de energia segundo Planck. Revista Brasileira de Ensino de Física 2000, 22, 523. [Link]

${ }^{8}$ Feldens, B.; Dias, P. M.; Santos, W. M. Let there be quantum. Revista Brasileira de Ensino de Física 2011, 32, 2602. [CrossRef]

${ }^{9}$ Del Regato, J. A. Max Planck. International Journal of Radiation Oncology Biology Physics 1979, 5, 2097. [CrossRef]

${ }^{10}$ Planck, M. On the law of distribution of energy in the normal spectrum. Annalen der Physik 1901, 4, 553. [Link]

${ }^{11}$ Volchan, S. B. A probabilidade na mecânica estatística clássica. Revista Brasileira de Ensino de Física 2006, 28, 313. [Link]
12 Barbosa, A. G. H. A estranha e contraditória relação entre os químicos e a química teórica. Revista Virtual de Química 2009, 1, 212. [CrossRef]

${ }^{13}$ Gearhart, C. A. Planck, the Quantum, and the Historians. Phisics in Pespective 2002, 4, 170. [Link]

${ }^{14}$ Freire Jr, O.; Pessoa Jr., O.; Bromberg, J. L. Em O itinerário de Louis de Broglie em busca de uma interpretação causal para a mecânica ondulatória, Santos, P. V. M., ed.; EDUEPB: Campina Grande, 2011. [Link]

${ }^{15}$ Freire Jr, O.; Pessoa Jr., O.; Bromberg, J. L. Em De Louis de Broglie a Erwin Schrödinger: uma comparação, Martins, R. A., ed.; EDUEPB: Campina Grande, 2011. [Link]

${ }^{16}$ Peixoto, E. M. A. Química Quântica. Parte I: O átomo de hidrogênio. Química Nova 1978, 1, 5. [Link]

${ }^{17}$ Simões, A. Max Planck (1858-1947), um revolucionário conservador. Gazeta de Física 2008, 31, 7. [Link]

${ }^{18}$ Moreira, I. C. 1905 um ano miraculoso. Ciência Hoje 2005, 36, 34. [Link]

${ }^{19}$ Pielawa, S.; Morigi, G.; Vitali, D.; Davidovich, L. Generation of EinsteinPodolsky-Rosen-Entangled Radiation through an Atomic Reservoir. Physical Review Letters 2007, 98, 240401. [CrossRef]

${ }^{20}$ Carvalho, S. H. M.; Einstein: uma luz sobre a luz. Ano Internacional de Física, 2005. 32p. [Link]

${ }^{21}$ Kleppner, D. Rereading Einstein on radiation. Revista Brasileira de Ensino de Física 2004, 27, 87. [CrossRef]

${ }^{22}$ Filho, E. S. Einstein: o homem que mudou o pensamento do homem. Perspectiva Filosófica, 2005, 2, p. 11. [Link]

${ }^{23}$ Braga, J. P, Filgueiras, C. A. L. The centennial of Bohr's Theory. Química Nova 2013, 36, 1073. [CrossRef]

${ }^{24}$ Del Regato, J. A. Niels Bohr. International Journal of Radiation Oncology Biology Physics 1981, 7, 509. [CrossRef]

${ }^{25} \mathrm{Li}$-Zhi, F. From the colapse of the atom to the colapse of the universe - Niels Bohr and Astrophysics. Chinese Astronomy and Astrophysics 1986, 8, 34. [CrossRef]

${ }^{26}$ Bohr, N.; The structure of the atom. Nobel Lecture, December 11, 1922. [Link] 
${ }^{27}$ McQuarrie, D. A.; Simon, J. D.; Physical Chemistry: A Molecular Approach, University Science Books, 1997.

${ }^{28}$ Ganci, S. Analytical and experimental remarks on the Sommerfeld's half planee solution. Optik Optics 2010, 121, 993 [CrossRef]

${ }^{29}$ Studart, N.; da Costa, R. C. T.; Moreira, I. C. Theodoro Ramos e os primórdios da física moderna no Brasil. Física na Escola 2004, 5, 34. [Link]

${ }^{30}$ Seth, S. Crafting the quantum: Arnold Sommerfeld and older quantum theory. Studies in History and Philosophy of Science 2008, 39, 335. [CrossRef]

${ }^{31}$ Morgon, N. H. The behavior of the electron: an analysis of the compton effect and the de broglie's relation. Química Nova 2008, 31, 1869. [CrossRef] 